\title{
Prevalence and Determinants of Pre-diabetes and Latent Tuberculosis Infection Among Apparently Healthy Adults in Three Communities in Southern Nigeria
}

\author{
Benson Olu Akinshipe ${ }^{1, \text { * }}$, Edirin Omorigho Yusuf ${ }^{2}$, Felix Oladapo Akinshipe ${ }^{3}$, \\ Muyiwa Adeleye Moronkeji ${ }^{4}$, Anthony Chukwuka Nwaobi ${ }^{5}$ \\ ${ }^{1}$ Departments of Medical Microbiology, School of Clinical Medicine, Igbinedion University \& Igbinedion University Teaching Hospital, \\ Okada, Nigeria \\ ${ }^{2}$ Department of Medical Microbiology, School of Clinical Medicine, University of Benin/Teaching Hospital, Benin City, Nigeria \\ ${ }^{3}$ Department of Internal Medicine, Obafemi Awolowo University Teaching Hospitals Complex, Ile-Ife, Nigeria \\ ${ }^{4}$ Department of Chemical Pathology, Ladoke Akintola University of Technology Teaching Hospital, Oshogbo, Nigeria \\ ${ }^{5}$ Department of Medical Laboratory Science, Igbinedion University, Okada, Nigeria
}

Email address:

bensonakinshipedr01@yahoo.com (B. O. Akinshipe)

${ }^{*}$ Corresponding author

\section{To cite this article:}

Benson Olu Akinshipe, Edirin Omorigho Yusuf, Felix Oladapo Akinshipe, Muyiwa Adeleye Moronkeji, Anthony Chukwuka Nwaobi. Prevalence and Determinants of Pre-diabetes and Latent Tuberculosis Infection Among Apparently Healthy Adults in Three Communities in Southern Nigeria. International Journal of Immunology. Vol. 7, No. 2, 2019, pp. 23-32. doi: 10.11648/j.iji.20190702.11

Received: July 3, 2019; Accepted: July 26, 2019; Published: August 14, 2019

\begin{abstract}
Type 2 diabetes mellitus (DM) is commonly associated with pulmonary tuberculosis (TB) and vice versa. Although most individuals with DM and TB pass through intermediate state of pre-diabetes mellitus (PDM) and latent tuberculosis infection (LTBI) respectively, however, data is scant on PDM and LTBI in co-endemic populations to inform recommendations on intervention in those settings. This study aimed to assess the prevalence of PDM and LTBI and determine the susceptibility factors among presumably low- risk adults in a high DM-and TB setting. A cross-sectional study of 352 ablebodied adults was conducted in three communities representative of the southern Nigeria population. Socio-demographic and anthropometric data of the participants were collected using a modified WHO STEPS Wise instrument. A single random blood sample was used to measure glycated hemoglobin $\left(\mathrm{HbA}_{1 \mathrm{c}}\right)$ using Cobas c111 Auto-analyzer and interferon-gamma (INF- $\gamma$ ) production with the aid of Quantiferon TB-Gold-In Tube (QFT-GIT) kit. Pre-diabetes was defined as HbA $1 \mathrm{c}$ between 5.7-6.4\% and LTBI as INF- $\gamma$ positivity $(\geq 0.35 \mathrm{IU} / \mathrm{ml})$. Overall, the prevalence of PDM was $29(8.2 \%)(95 \%$ CI: 6.4-9.4\%) and LTBI 83 (23.6\%) (95\% CI: 21.6-27.3\%), while 9 (2.6\%) had dual PDM-LTBI. The urbanites recorded higher PDM 15 (12.8\%) $\left(X^{2}=6.340, p=0.022\right)$ whereas LTBI was higher among villagers $48(43.6 \%)\left(X^{2}=36.503, p=0.0001\right)$. Risk factors associated with PDM and LTBI were: residence status, aging, smoking, familial DM and non-BCG vaccination. Aging was the single most important predictor of PDM $\left(X^{2}=8.469, p=0.007\right)$, LTBI $\left(X^{2}=59.541, p=0.001\right)$; with aged 50-59 years having four-fold higher risk of PDM-LTBI $(\mathrm{OR}=4.72,95 \% \mathrm{CI}=3.25 ; \mathrm{p}=0.0001)$. These findings indicate that one in twelve and one in four presumably healthy persons screened were found to harbor PDM and LTBI respectively. There is a twin epidemic of PDM and LTBI among southern Nigeria adults. Susceptibility pattern suggests environmental, familial and cumulative life-time risks. 'Syndemic' DM and TB in the area is imminent in the absence of timeous intervention.
\end{abstract}

Keywords: Adults, Communities, Pre-diabetes, Latent Tuberculosis Infection, Risk Factors

\section{Introduction}

Concurrent type-2 diabetes mellitus (DM) and pulmonary tuberculosis (TB) is one of the most common co-morbid and mortal conditions worldwide. Available reports indicate that more than $70 \%$ of patients with DM live in the low - and 
middle- countries (LMICs) and that up to $95 \%$ of TB patients also live in the same countries [1,2]; where the prevalence of these diseases continues to increase in parallel [3]. The harmful confluence of these two chronic disorders along with its attendant immunosuppressive effects has been noted as one of the most costly and burdensome health problems of the present times [4, 5]. The realization of the dire consequences of the convergence of diabetes and tuberculosis diseases prompted the World Health Organization (WHO) and International Union Against Tuberculosis and Lung Disease (IUATLD) to launch The Collaborative Framework on Diabetes and Tuberculosis Co-morbidity in order to encourage studies to better understand the interrelationship between the two diseases and to inform planning and implementation for prevention, care and control of DM-TB co-morbidity [6]. The reason for the increased risk of TB in DM and vice-versa is not yet clear.

In the majority of cases, both DM and TB are preceded by asymptomatic latent preliminary stage, pre-diabetes mellitus (PDM) and latent tuberculosis infection (LTBI) respectively. These two conditions can co-exist undetected for many years $[7,8]$, causing latent irreversible damage to vital body organs [9]. Pre-diabetes and latent tuberculosis infection represent the tip of the iceberg respectively, for diabetes and tuberculosis disease.

Pre-diabetes or intermediate hyperglycemia recognized as an intermediate phase in the natural history of DM is associated with poor glycemic control, and is a high risk state for DM that is defined by glycemic variables that are higher than normal but lower than diabetic threshold; and with an annualized conversion rate of $5-10 \%$ [10]. The conversion rate from pre-diabetic to diabetic would vary by population characteristics and individual lifestyle and /or environmental risk factors. The WHO/International Diabetic Association (IDF) (WHO/IDF) criteria for diagnosing PDM are fasting plasma glucose level of between 6.1 and $6.9 \mathrm{mmol} / \mathrm{L}$ [11]. The use of glycated hemoglobin $\left(\mathrm{HbA}_{1 \mathrm{c}}\right)$ has been agreed upon as the most important biochemical indicator of long-term glycemic control in dysglycemic individuals [12]. An important advantage of the $\mathrm{HbA}_{1 \mathrm{c}}$ test is that the blood sample can be drawn at any time, because it is not affected by short time variations, such as food intake, exercise and stress. Glycated hemoglobin levels of between 5.7 - 6.4\% has been introduced as a more precise measure for PDM category of high diabetes risk in adults [10, $13,14]$. Pre-diabetes mellitus is recognized as an independent risk factor for developing TB [15].

Latent tuberculosis infection (LTBI) is a state in which persistent immune response occurs to Mycobacterium tuberculosis $(\mathrm{MTb})$ without evidence of manifestation of clinical or other symptoms of active TB disease. Approximately one third of the World's population is estimated to be harboring LTBI and are at risk of TB reactivation; and a vast pool of individuals with LTBI persists in developing countries, posing a major barrier to global TB control [16]. A weak immune system increases the risk of progression from LTBI to active disease. This could be particularly evident in high risk groups or vulnerable subpopulations, such as those with concurrent conditions, including PDM/DM. The lifetime risk of progressing from LTBI with DM is $30 \%$ compared to the $5-10 \%$ estimated previously without it [17]; the progression risk is one of the focuses of most TB control strategies. Screening and managing individuals for LTBI could minimize the risk of progression to active TB. Currently, it is not possible to directly identify LTBI in humans. Latent tuberculosis infection is diagnosed by detecting memory $\mathrm{T}$ cell response against latent infection with highly specific Mycobacterium tuberculosis antigens using Interferon- gamma (INF- $\gamma$ ) Release Assays (IGRAs), such as the Quantiferon- TB GoldIn- Tube (QFT-GIT) enzyme-linked immune-sorbent assay (ELISA) [18].

There is an increasing prevalence of pre-diabetes and diabetes worldwide [19]. In recent decades, TB incidence has declined in high-income countries, but incidence remains high in countries that have high rate of DM [20]. Recent studies have shown an increased prevalence of diabetes in people with TB, especially in the LMICs. For instance, Nigeria, the most populous country in Africa and the current leader in the number of TB cases and currently rated $6^{\text {th }}$ globally among the 22 high TB-burden countries (HBCs) [20], has been noted to be facing an increasing rate of DM among its population [21]. Diabetes is the most common metabolic disorder in Nigeria, and it has been observed that its prevalence has been rising at an alarming rate in the last two decades in the country [21]; this carries with it a concomitant increase in the prevalence of TB.

The emerging convergence of DM and TB epidemics in Nigeria and its associated complications resulting in increased morbidity and mortality raises the question of how best to control the impending twin epidemics in the country. There is currently limited data to inform recommendations on intervention in order to alleviate the DM- TB burden in resource-limited co-endemic populations.

Pre-diabetes mellitus and LTBI as asymptomatic forerunners of diabetes and tuberculosis respectively, are mostly detected on routine screening in apparently healthy people. Accurate detection of PDM and LTBI and identification of co-existing risk factors, and appropriate treatment of probable cases-to-be could reduce the risk of progression to the corresponding overt disease, and indeed of the co-morbid DM-TB condition, and increase case detection rates. However, surveys for these precursor stages are rarely performed in non-high risk groups, especially in co-endemic populations; and there is, therefore, a knowledge gap regarding these latent conditions and their correlates in those settings.

As part of DM-TB control program, this study set out to simultaneously assess the prevalence of pre-diabetes and latent tuberculosis infection, using systematic screening methods, in a convenient sample of a cross-section of apparently healthy adults from three different geographic communities, representative of the southern Nigeria population. The study also aimed to identify associated risk factors predisposing the residents to pre-diabetes and latent tuberculosis infection. 


\section{Participants and Methods}

\subsection{Settings and Participant Selection}

This was a multi-community cross-sectional study carried out from September to December 2018. The study involved able-bodied female and male adult volunteers randomly recruited from a cross-section of three different geographic sites, namely: Usen village (rural), Okada town (semi-urban) and Benin City (urban), all located in Edo state, southern Nigeria. Usen community is a rural, mainly agrarian settlement situated $7.5 \mathrm{kms}$ from Okada. Okada 'university town', the administrative headquarters of Ovia North East Local Government Area, Edo state, Nigeria is home to Igbinedion University and Igbinedion University Teaching Hospital. Benin City, located about 45 minutes driving distance from Okada, is one of the largest cities in southern Nigeria and doubles as the capital city of Edo state. Benin City is where both the University of Benin, Nigeria and the University of Benin Teaching Hospital are located. These three sites were chosen for the current study primarily because of convenience and their contiguity, and in order to capture a representative sample of homes from all three different geographic communities according to the classification of the latest Nigeria's National Bureau of Statistics [22]; these heterogeneous communities are composed of individuals originally from different Nigeria ethnic groups.

Four criteria were used in selecting the participants in this study. These were that (1) subject aged $\geq 20$ years at the time of the study and provided consent, (2) the person must not be a known diabetic patient diagnosed for 12 weeks or less prior to this study, with need for injected insulin therapy as per standard Nigeria Diabetic Society (NDS) guidelines [23], (3) the individual must not be positive for $\mathrm{TB}$ in chest radiographs or bacteriological-culture confirmed TB patient on anti-TB treatment and evaluated according to the Nigeria National Tuberculosis and Leprosy and Buruli Ulcer Control Program (NTLCP) guidelines [24], and (4) participant must not be an immunosuppressed person (such as HIV/AIDS, on immunosuppressant drug (s) or pregnant women). Exclusion criteria focused on medical conditions that would potentially confound results or interfere with save completion of the study, including metabolic disorders, active infections, hemoglobinopathies, pregnancy and recent participation in a clinical trial.

Ethics: The primary study was approved by the Igbinedion University Okada/ Igbinedion University Teaching Hospital (IUO/IUTH) Research and Ethics Institutional Review board and permission was granted by the Public Health authorities, the Chiefs and Community Leaders of the study sites prior to the commencement of this study. Oral and written information in English language was provided to study participants before consent was obtained. The confidentiality of study participants was fully respected and the analyses performed did not identify any participants; the clinical samples were coded. Study volunteers who were found to have $\mathrm{DM}$ and/or TB were referred to the metabolic and TB clinics respectively, for counselling, further investigation and appropriate follow-up treatment and management.

\subsection{Data Collection and Physical Assessments}

After giving written informed consent, each of 364 selected volunteers from the three sites was subjected to paper-based pre-designed interviewer-administered risk screening, partially adapted from the generic WHO STEPS Wise instrument approach for collecting surveillance data [25]; it was modified to and tailored to suit Nigeria environment through review of relevant literature. The pretested Questionnaire Interview tool was standardized across all the three study sites and administered by trained bilingual (English and local Language-speaking) data-collectors at respondents' residences; the local language was used to explain verbally to the participants who could not understand the medical language in the questionnaire. Information gleaned from subjects included selected social, demographic and behavioral variables (residence, age, sex, marital status, level of formal education attained, occupation, housing index, smoking habits, alcohol consumption and level of physical activity). Participants were classified into those that met the WHO minimum recommendations for physical activity (at least 75 minutes of vigorous-intensity, or 150 minutes of moderate-intensity activities per week) and those that did not, and as described elsewhere [26]. In addition, family history of raised blood glucose (diabetes) and treatment or diabetes evaluation and of tuberculosis among first degree relatives, history of hypertension and/or antihypertensive medication were collected. Hypertension was defined as having a systolic and/or diastolic blood pressure $\geq$ 140/90 $\mathrm{mm} \mathrm{Hg}$ or documented use of anti-hypertensive (s) in a previously diagnosed person with hypertension, or a selfreporting hypertension. Participants identified to be hypotensive were classified as non-hypertensive for the purpose of this study. Some of the questions demanded 'Yes'/ 'No' answer. Responses of "don't know" or "refused" were recorded as missing for all variables. Standardized approaches as detailed elsewhere [27] were used at all sites for anthropometric measurements, viz.: height, weight, abdominal/waist circumference, and for the calculation of body mass index (BMI).

Information supplied were cross-checked with the medical records at the respective local health center. BacilleCalmette-Guerin (BCG) vaccination was confirmed by the presence of BCG scar (s).

\subsection{Blood Processing and Analyses}

About 7-8ml of venipuncture-collected whole-blood sample was obtained aseptically from the ante-cubital vein of each study participant. A $2 \mathrm{ml}$ aliquot of the blood sample was transferred into ethylene di-amine tetra acetic acid (EDTA) vacuitainer, mixed gently and used for the estimation of hemoglobin $(\mathrm{Hb})$ level using the Automated Hematology Analyzer (Sysmex ICX-21N) and for CD4+T lymphocyte counts with the aid of the Fluorescence 
Activated Cell Sorter (FACS) Auto-analyzer (BectonDickinson, Kaplan Scientific Inc., Japan). A $2 \mathrm{ml}$ of the anticoagulant free blood sample was allowed to clot for $2 \mathrm{hrs}$ at room temperature $\left(\mathrm{RT}^{0}\right)$ before centrifugation at $5000 \mathrm{rpm}$ for $10 \mathrm{mins}$ to obtain serum. The harvested sera were stored at $20^{\circ} \mathrm{C}$ until they were tested. The stored sera were thawed out at $\mathrm{RT}^{0}$ before testing in batches of 50 samples per assay for the clinical chemistry immunoassays using the Cobas c111 System Auto-analyzer (Roche/Hitachi Diagnostics) [28]. The biochemical parameters analyzed were the levels of namely: glycated hemoglobin $\left(\mathrm{HbA}_{1 \mathrm{c}}\right)$; serum C-reactive protein (CRP); the lipoproteins, viz.: Total cholesterol, Low Density Lipoprotein (LDL-C3) and High Density Lipoprotein (HDL$\mathrm{C} 4)$. The remaining blood sample $(3 \mathrm{ml})$ was drawn into heparinized tube and the extracted plasma used to test for INF- $\gamma$ production by the sandwich ELISA with the aid of the Quantiferon-TB Gold In-Tube (QFT-GIT) test Kit (Cellestis Ltd., Carnegie, Australia) [29], and as previously described by Akinshipe et al. [30]. The calculation of the results of INF- $\gamma$ released was based on the QFT-GIT Analysis Software Program provided by Kit manufacturer (Cellestis Ltd., Carnegie, Australia).

\subsection{Quality Controls and Cut-off Points}

To assure representativeness of the southern Nigeria adult population, study participants were recruited from crosssection of three different and heterogeneous communities. To ensure the quality of the data supplied by participants, the questionnaire instrument was pre-tested and standardized across the three sites; the answers were thoroughly checked for completeness and consistency, cleaned and computerrecorded by study supervisors who were not involved in the collection. PreciControl standards using Calibrator for Automated Systems (c. f. a. s) were employed to maintain the quality of blood measurements. The $\mathrm{HbA}_{1 \mathrm{c}}$ levels for prediabetes detection were determined under the auspices of the Nigeria's National Glycohemoglobin Standardization Program (NGSP) Reference Laboratory (Ladoke Akintola University of Technology Teaching Hospital, Oshogbo, Nigeria). Pre-diabetes classification was according to the revised WHO criteria and studies, which suggested $\mathrm{HbA}_{1 \mathrm{c}}$ cut-off points of $5.7-6.4 \%[11,12,13]$. In the INF- $\gamma$ ELISA runs, the variation of the inter-plate control did not generally exceed $10 \%$. The cytokine result was considered positive when the level of MTb antigen was $\geq 0.35 \mathrm{IU} / \mathrm{ml}$ and $\geq 25 \%$ of the Nil tube. Negative control concentration of at least 8.0 $\mathrm{IU} / \mathrm{ml}$ and positive difference value of less than $0.5 \mathrm{IU} / \mathrm{ml}$ was considered "indeterminate" (based on the manufacturer's guidelines). The presence of LTBI was determined by a positive QFT-GIT test result that adjusted for background noise, heterophile antibody effects, or non-specific INF- $\gamma$ samples. Participants with indeterminate QFT-GIT test results were excluded from the study data because the test could not be repeated since test had deployed.

\subsection{Data Management and Analysis}

The demographic, lifestyle, anthropometric and clinical laboratory raw data for the study participants were collated with the aid of Microsoft Access Database (Microsoft Corp., Redmond, Australia). Results are given as means \pm Standard deviation (SD), frequencies or percentages for categorical variables. Prevalence was defined as the number of individuals with the respective condition or disease divided by the total number of individuals in a cross-sectional, population-based screening. Student's t tests, Pearson's chi square tests, Fisher's exact and McNemar-Bowker tests were used to assess association based on each factor and to compare differences between groups and for binary outcome variables. In addition, binomial logistic regression analyses were used to compute Odds ratio (OR) and corresponding weighted prevalence, or $95 \%$ confidence interval (CI) and to assess risk factors associated with or independent of prediabetes and/ or latent tuberculosis infection. Statistical analysis was performed using Statistical Package for Social Sciences (SPSS), version 20.0 Software (SPSS Inc., Chicago, IL., USA) for windows. P values $\leq 0.05$ were considered statistically significant for all analysis.

\section{Results}

Out of 374 able-bodied adults originally recruited for this study, $362(96.8 \%)$ were eligible and consented to participate. Ten of the participants were excluded from the final results: three individuals had incomplete /missing data, two persons each had undiagnosed diabetes and tuberculosis respectively, one had undiscovered concurrent diabetes and tuberculosis and two subjects recorded indeterminate INF- $\gamma$ test result. Overall, a final total of $352(94.1 \%)$ of the recruits were enrolled and included in the study analysis.

\subsection{Participants Socio-demographics and Anthropometrics}

The demographic, behavioral and anthropometric characteristics of the 352 enrolled participants stratified according to residence status are presented in Table 1. The site-distribution of the enrollees was: villagers $110(31.3 \%)$, semi-urbanites 125 (35.5\%), urbanites 117 (33.2\%). Across all the sites, almost equal number of females and males participated, the majority were aged $\geq 40$ yrs. (mean $=51.32 \pm 12.27$ yrs.); and most reported been married or cohabiting with a partner as at the time of this study. Other socio-demographic characteristics of the enrollees are shown in Table 1. Equally high proportion $(91.2 \%)$ of the subjects reported no family history of diabetes or pulmonary tuberculosis, while majority were not diagnosed to be hypertensive. Obesity was fairly common, especially among the urban dwellers. A high majority $331(94.0 \%)$ of the adults had received BCG vaccination as evidenced from scar (s) or vaccination records. 
Table 1. Socio-demographic and Anthropometric Characteristics of enrolled Participants Stratified by Site.

\begin{tabular}{|c|c|c|c|c|c|c|}
\hline Variable $^{\mathrm{a}}$ & Rural n=110 & Sub Urban $n=125$ & Urban $n=117$ & Overall n (\%) & $\mathrm{X}^{2}$-values & p-value \\
\hline \multicolumn{7}{|l|}{ Sex } \\
\hline Male & $62(56.4)$ & $55(44.0)$ & $65(55.6)$ & $182(51.7)$ & & \\
\hline Female & $48(43.6)$ & $70(56.0)$ & $52(44.4)$ & $170(48.3)$ & 4.623 & 0.099 \\
\hline \multicolumn{7}{|l|}{ Age group (Years) } \\
\hline$<40$ & $17(15.5)$ & $34(27.2)$ & $29(24.8)$ & $80(22.7)$ & & \\
\hline $40-49$ & $23(20.9)$ & $30(24.0)$ & $46(39.3)$ & $99(28.1)$ & & \\
\hline $50-59$ & $32(29.1)$ & $35(28.0)$ & $22(18.8)$ & $89(25.3)$ & & \\
\hline \multicolumn{7}{|l|}{ Marital Status } \\
\hline Married & $95(86.4)$ & $106(84.8)$ & $91(77.8)$ & $292(83.0)$ & & \\
\hline Single & $4(3.6)$ & $10(8.0)$ & $18(15.4)$ & $32(9.1)$ & & \\
\hline Widowed & $11(10.0)$ & $9(7.2)$ & $8(6.8)$ & $28(8.0)$ & 10.296 & $0.036^{*}$ \\
\hline \multicolumn{7}{|l|}{ Level of Education } \\
\hline Primary & $53(48.2)$ & $17(13.6)$ & $17(14.5)$ & $87(24.7)$ & & \\
\hline Secondary & $38(34.5)$ & $53(42.4)$ & $42(35.9)$ & $133(37.8)$ & & \\
\hline Unemployed & $7(6.4)$ & $8(6.4)$ & $11(9.4)$ & $26(7.4)$ & & \\
\hline Unskilled Worker & $38(34.5)$ & $31(24.8)$ & $25(21.4)$ & $94(26.7)$ & & \\
\hline Skilled Worker & $12(10.9)$ & $37(29.6)$ & $28(23.9)$ & $77(21.9)$ & & \\
\hline Business/Professional & $18(16.4)$ & $28(22.4)$ & $40(34.2)$ & $86(24.4)$ & & \\
\hline Retired/Housewife & $35(31.8)$ & $21(16.8)$ & $13(11.1)$ & $69(19.6)$ & 35.476 & $0.001 *$ \\
\hline \multicolumn{7}{|l|}{ Crowing index } \\
\hline 1-4 person/House & $19(17.3)$ & $53(42.4)$ & $35(29.9)$ & $107(30.4)$ & & \\
\hline$\geq 5$ Person/House & $91(82.7)$ & $72(57.6)$ & $82(70.1)$ & $245(69.6)$ & 17.480 & $0.001 *$ \\
\hline \multicolumn{7}{|l|}{ Smoking Status } \\
\hline Never & $97(88.2)$ & $115(92.0)$ & $109(93.2)$ & $321(91.2)$ & & \\
\hline Current & $7(6.4)$ & $6(4.8)$ & $5(4.3)$ & $18(5.1)$ & & \\
\hline Former & $6(5.5)$ & $4(3.2)$ & $3(2.6)$ & $13(3.7)$ & 2.100 & 0.717 \\
\hline \multicolumn{7}{|l|}{ Alcoholism } \\
\hline No & $106(96.4)$ & $118(94.4)$ & $102(87.2)$ & $326(92.6)$ & & \\
\hline Yes & $4(3.6)$ & $7(5.6)$ & $15(12.8)$ & $26(7.4)$ & 7.895 & $0.019 *$ \\
\hline Yes & $91(82.7)$ & $80(64.0)$ & $64(54.7)$ & $235(66.8)$ & 20.735 & $0.001^{*}$ \\
\hline \multicolumn{7}{|l|}{ Family History of Diabetes } \\
\hline No & $104(94.5)$ & $115(29.0)$ & $102(87.2)$ & $321(91.2)$ & & \\
\hline Yes & $6(5.5)$ & $10(8.0)$ & $15(12.8)$ & $31(8.8)$ & 3.987 & 0.136 \\
\hline \multicolumn{7}{|l|}{ Family History of TB } \\
\hline No & $104(94.5)$ & $115(29.0)$ & $102(87.2)$ & $321(91.2)$ & & \\
\hline Yes & $6(5.5)$ & $10(8.0)$ & $15(12.8)$ & $31(8.8)$ & 3.987 & 0.136 \\
\hline \multicolumn{7}{|l|}{ Hypertension } \\
\hline No & $92(83.6)$ & $103(82.4)$ & $80(68.4)$ & $275(78.1)$ & & \\
\hline Yes & $18(16.4)$ & $22(17.6)$ & $37(31.6)$ & $77(21.9)$ & 28.351 & $0.001 *$ \\
\hline \multicolumn{7}{|l|}{ Body Mass Index $\left(\mathrm{kg} / \mathrm{m}^{2}\right)$} \\
\hline Underweight $(\leq 18.5)$ & $4(3.6)$ & $2(1.6)$ & $2(1.7)$ & $8(2.3)$ & & \\
\hline Normal (18.5-24.9) & $86(78.2)$ & $59(47.2)$ & $43(36.8)$ & $188(53.4)$ & & \\
\hline Overweight/Obese $(\geq 25.0)$ & $20(18.2)$ & $64(51.2)$ & $72(61.5)$ & $156(44.3)$ & 47.051 & $0.001 *$ \\
\hline \multicolumn{7}{|l|}{ Waist circumference } \\
\hline Normal & $79(71.8)$ & $73(58.4)$ & $36(30.8)$ & $168(47.7)$ & & \\
\hline Abdominal obesity & $31(28.2)$ & $52(41.6)$ & $81(69.2)$ & $184(52.3)$ & 40.322 & $0.001 *$ \\
\hline \multicolumn{7}{|l|}{ BCG Vaccinated } \\
\hline No & $8(73)$ & $8(6.4)$ & $5(4.3)$ & $21(6.0)$ & & \\
\hline Yes & $102(92.7)$ & $117(93.6)$ & $112(95.7)$ & $331(94.0)$ & 7.75 & $0.017 *$ \\
\hline
\end{tabular}

DM-Diabetes mellitus; TB-Tuberculosis; Kg-Kilogram

${ }^{a}$ Categorical variables are reported as number of participants and percentage in parenthesis

Chi square test for $\mathrm{p}$ values; $* \mathrm{p} \leq 0.05$ was considered significant

\subsection{Clinical Laboratory Measurements}

The results of selected blood parameters for the participants stratified by residence status are presented in Table 2. There were no significant differences among the participants from the three communities with respect to the mean CD4+T lymphocyte counts, hemoglobin concentrations and C-reactive protein profiles, although there were individual variation within the groups (results not shown). There was significant difference $(p=0.001)$ in the total cholesterol, but not in respect to LDL-C3 and HDL-C4 levels 
among the site-groups; however, the rural dwellers' mean HDL-C4 level (43 $\pm 5.2 \mathrm{mg} / \mathrm{dl})$ was relatively higher but not significantly different from the corresponding levels recorded for the semi-urban and urban residents respectively, $\mathrm{p}=0.08$.

The blood CD4+T lymphocyte counts, hemoglobin concentrations, C-reactive protein and cholesterol levels of the participants were observed to be generally within the normal reference ranges as determined for healthy adult Nigerians and published elsewhere [31].

Table 2. Laboratory data of the healthy adult participants according to residence status.

\begin{tabular}{|c|c|c|c|c|}
\hline Parameter & Rural (n=110) & Semi Urban $(n=125)$ & Urban $(n=117)$ & $P$ value \\
\hline CD4+ counts \pm SD cells $/ \mu \mathrm{l}$ & $825 \pm 127.8$ & $838.9 \pm 102.6$ & $849.2 \pm 72.9$ & 0.21 \\
\hline $\mathrm{Hb}$ level mean $\pm \mathrm{SD}(\mathrm{g} / \mathrm{dl})$ & $13.3 \pm 0.2$ & $13.6 \pm 0.5$ & $13.9 \pm 0.3$ & 0.38 \\
\hline C-Reactive Protein mean $\pm \mathrm{SD}(\mathrm{mg} / \mathrm{L})$ & $1.6 \pm 0.08$ & $1.5 \pm 0.05$ & $1.8 \pm 0.06$ & 0.22 \\
\hline Total Cholesterol mean $\pm \mathrm{SD}(\mathrm{mg} / \mathrm{dl})$ & $145 \pm 15.7$ & $165 \pm 10.2$ & $186 \pm 12.5$ & $0.001 *$ \\
\hline LDL-C3Cholesterol mean \pm SD (mg/dl) & $93 \pm 10.4$ & $97 \pm 8.7$ & $101 \pm 13.2$ & 0.67 \\
\hline HDL-C4Cholesterol mean \pm SD (mg/dl) & $43 \pm 5.2$ & $38 \pm 4.6$ & $36 \pm 6.2$ & 0.08 \\
\hline
\end{tabular}

SD-Standard deviation; Hb-hemoglobin; LDL-C3-Low Density Lipoprotein; HDL-C4 -High Density Lipoprotein

$*$ p value $\leq 0.05$ was considered statistically significant.

\subsection{Prevalence of Pre-diabetes and Latent Tuberculosis Infection}

Figure 1 summarizes the prevalence of PDM and LTBI among the screened adults across the three Nigerian sites. The overall prevalence of pre-diabetes was $29(8.2 \%)(95 \%$ CI: $6.4-9.4 \%)$ and the prevalence of LTBI was $86(23.6 \%)$ (95\% CI: $21.6-27.3 \%$ ). The prevalence of PDM was highest among urban residents $15(12.8 \%)$ compared to semi-urban dwellers $10(8.0 \%)$ and rural residents $4(3.6 \%)$; $\left(\mathrm{X}^{2}=6.340\right.$, $\mathrm{df} 2, \mathrm{p}=0.022$ ). Significant difference was also observed in
LTBI for the urban participants $14(12.0 \%)$ compared with semi-urban dwellers and the rural residents who recorded 21 $(16.8 \%)$ and $48(43.6 \%)$ respectively $\left(X^{2}=36.503, \mathrm{df} 2\right.$; $\mathrm{p}=0.0001$ ).

This study observed that $9(2.6 \%)$ of the adults had dual PDM and LTBI (PDM-LTBI) (results not shown). Further analysis of the data revealed that the prevalence of PDM among adults with LTBI was more than twice the prevalence of those without LTBI.

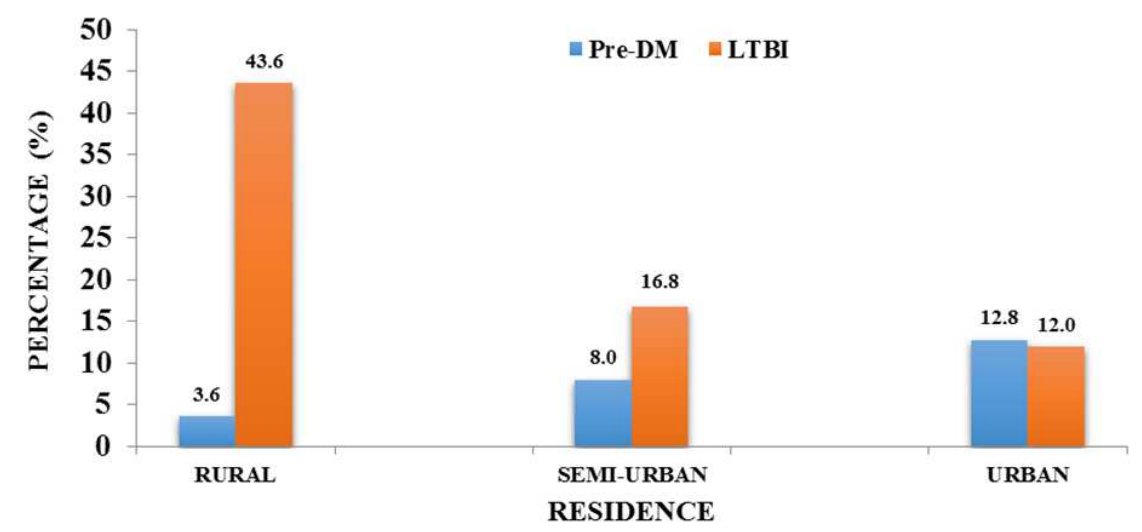

Figure 1. Site-specific Prevalence of Pre-diabetes and Latent Tuberculosis infection in Adults (N=352).

Pre-DM - Pre-Diabetes Mellitus: Glycated hemoglobin $\left(\mathrm{HbA}_{1 \mathrm{c}}\right)$ level of 5.7-6.4 and never been diagnosed of diabetes mellitus

LTBI - Latent tuberculosis: Quantiferon-TB Gold-in Tube (QFT-GIT) positive ( $\geq 0.35 \mathrm{IU} / \mathrm{ml})$ and never been diagnosed of tuberculosis

\subsection{Prevalence of PDM and LTBI and Determinants}

The association between the socio-demographic and anthropometric characteristics (variables) and the prevalence of PDM and LTBI among the participants is presented in Table 3. Bivariate analysis showed that age $\left(X^{2}=8.469\right.$, $\mathrm{p}=0.007)$, smoking $\left(\mathrm{X}^{2}=115.326, \mathrm{p}=0.001\right)$, family history of diabetes $\left(\mathrm{X}^{2}=13.131, \quad \mathrm{p}=0.002\right), \quad$ abdominal obesity $\left(X^{2}=8.469, p=0.004\right)$, hypertension $\left(X^{2}=15.972, p=0.001\right)$ and non-BCG vaccination $\left(\mathrm{X}^{2}=13.131, \mathrm{p}=0.002\right)$ were significantly associated with PDM. Similarly, age $\left(X^{2}=59.541, p=0.001\right)$, level of formal education $\left(X^{2}=10.640\right.$, $\mathrm{p}=0.005)$, smoking $\left(\mathrm{X}^{2}=44.698, \mathrm{p}=0.001\right)$, family history of DM $\quad\left(X^{2}=36.793, \quad p=0.0001\right), \quad$ family history of TB
$\left(\mathrm{X}^{2}=16.142, \quad \mathrm{p}=0.0001\right)$ and non-BCG vaccination $\left(X^{2}=28.375, p=0.001\right)$ were found to be significantly associated with LTBI. In the present study, gender, marital status, occupation, alcoholism, level of physical activity and BMI were not found to be significantly associated with either PDM or LTBI. However on logistic regression analysis age, smoking, family history of diabetes and non-BCG vaccination remained statistically significant for both conditions; with age $\geq 40$ years $(p=0.0002)$ and non-BCG vaccination $(p=0.001)$ being the most important predictors of concurrent PDM and LTBI (Table 4). In the determination of age-specific risk, older adults aged 50-59 years had significantly higher proportion of PDM-LTBI compared to 
the other age groups, $(\mathrm{OR}=4.72,95 \% \mathrm{CI}=3.25 ; \mathrm{p}=0.0001)$. Adults aged 50-59 years were found to be almost four times more likely to have PDM-LTBI compared to the younger 20-
39 years old category, while BCG vaccination confer almost two and half times protection against PDM-LTBI.

Table 3. Association between varying factors and Prevalence of PDM and LTBI among Participants $(N=352)$.

\begin{tabular}{|c|c|c|c|c|c|c|c|}
\hline \multirow{2}{*}{ Variable } & \multirow{2}{*}{$\mathbf{n}$} & \multicolumn{3}{|l|}{ PDM } & \multicolumn{3}{|l|}{ LTBI } \\
\hline & & Positive (\%) & Negative (\%) & p-value & Positive (\%) & Negative (\%) & p-value \\
\hline Overall population & 352 & $29(8.2)$ & $323(91.8)$ & & $83(23.6)$ & $269(76.4)$ & \\
\hline \multicolumn{8}{|l|}{ Sex } \\
\hline Male & 182 & $12(6.6)$ & $170(93.4)$ & & $47(25.8)$ & $135(74.2)$ & 0.305 \\
\hline Female & 170 & $17(10.0)$ & $153(90.0)$ & 0.245 & $36(21.2)$ & $134(78.8)$ & \\
\hline \multicolumn{8}{|l|}{ Age group (Years) } \\
\hline$<40$ & 80 & $3(3.8)$ & $77(96.3)$ & & $10(12.5)$ & $70(87.5)$ & \\
\hline $40-49$ & 99 & $6(6.1)$ & $93(94.0)$ & & $19(19.2)$ & $80(80.8)$ & \\
\hline $50-59$ & 89 & $15(16.9)$ & $74(83.1)$ & & $47(52.8)$ & $42(47.2)$ & \\
\hline$\geq 60$ & 84 & $5(6.0)$ & $79(94.0)$ & $0.007 *$ & $7(8.3)$ & $77(91.7)$ & $0.001 *$ \\
\hline \multicolumn{8}{|l|}{ Marital Status } \\
\hline Married & 292 & $24(8.2)$ & $268(91.8)$ & & $63(21.6)$ & $229(78.4)$ & \\
\hline Single & 32 & $3(9.4)$ & $29(90.6)$ & & $11(34.4)$ & $21(65.6)$ & \\
\hline Widowed & 28 & $2(7.1)$ & $26(92.9)$ & 0.952 & $9(32.1)$ & $19(67.9)$ & 0.145 \\
\hline Primary & 87 & $9(10.3)$ & $78(89.7)$ & & $25(28.7)$ & $62(71.3)$ & \\
\hline Secondary & 133 & $8(6.0)$ & $125(94.0)$ & & $27(20.3)$ & $106(79.7)$ & \\
\hline Tertiary & 132 & $12(9.1)$ & $120(90.9)$ & 0.314 & $31(23.5)$ & $101(76.5)$ & $0.005^{*}$ \\
\hline \multicolumn{8}{|l|}{ Occupation Group } \\
\hline Unemployed & 51 & $4(7.8)$ & $47(92.2)$ & & $13(25.5)$ & $38(74.5)$ & \\
\hline Unskilled Worker & 86 & $5(5.8)$ & $81(94.2)$ & & $18(20.9)$ & $68(79.1)$ & \\
\hline Skilled Worker & 70 & $3(4.3)$ & $67(95.7)$ & & $15(21.4)$ & $55(78.6)$ & \\
\hline Business/Professional & 76 & $8(10.5)$ & $68(89.5)$ & & $16(21.1)$ & $60(78.9)$ & \\
\hline Retired/Housewife & 69 & $9(13.0)$ & $60(87.0)$ & 0.137 & $21(30.4)$ & $48(69.6)$ & 0.612 \\
\hline \multicolumn{8}{|l|}{ Smoking Status } \\
\hline Never & 321 & $11(3.4)$ & $310(96.6)$ & & $61(19.0)$ & $260(81.0)$ & \\
\hline Current & 18 & $9(50.0)$ & $9(50.0)$ & & $11(61.1)$ & $7(38.9)$ & \\
\hline Former & 13 & $9(69.2)$ & $4(30.8)$ & $0.001 *$ & $11(84.6)$ & $2(15.4)$ & $0.001 *$ \\
\hline \multicolumn{8}{|l|}{ Alcoholism } \\
\hline No & 326 & $27(8.3)$ & $299(91.7)$ & & $80(24.5)$ & $246(75.5)$ & \\
\hline No & 235 & $24(10.2)$ & $211(89.8)$ & & $49(20.9)$ & $186(79.1)$ & \\
\hline Yes & 117 & $5(4.3)$ & $112(95.7)$ & 0.056 & $34(29.1)$ & $83(70.9)$ & 0.363 \\
\hline \multicolumn{8}{|c|}{ Family History of Diabetes } \\
\hline No & 321 & $22(6.9)$ & $299(93.1)$ & & $62(19.3)$ & $259(80.7)$ & \\
\hline Yes & 31 & $7(22.6)$ & $24(79.4)$ & $0.002 *$ & $21(67.7)$ & $10(32.3)$ & $0.0001 *$ \\
\hline \multicolumn{8}{|l|}{ Family History of TB } \\
\hline No & 337 & $27(8.0)$ & $310(92.0)$ & & $73(21.7)$ & $264(78.3)$ & \\
\hline Yes & 15 & $2(13.3)$ & $10(86.7)$ & 0.705 & $10(66.7)$ & $5(33.3)$ & $0.0001 *$ \\
\hline \multicolumn{8}{|c|}{ Body Mass Index $\left(\mathrm{kg} / \mathrm{m}^{2}\right)$} \\
\hline Underweight $(<18.5)$ & 8 & $1(12.6)$ & $7(87.5)$ & & $2(25.0)$ & $6(75.0)$ & \\
\hline Normal (18.5-24.9) & 188 & $10(5.3)$ & $178(94.7)$ & & $46(24.5)$ & $142(75.5)$ & \\
\hline Overweight/Obese & 156 & $18(11.5)$ & $138(88.5)$ & 0.102 & $35(22.4)$ & $121(77.6)$ & 0.391 \\
\hline \multicolumn{8}{|l|}{ Waist circumference } \\
\hline Normal & 168 & $8(4.8)$ & $160(95.2)$ & & $48(28.6)$ & $120(71.4)$ & \\
\hline Abdominal obesity & 184 & $21(11.4)$ & $164(88.6)$ & $0.004 *$ & $35(19.0)$ & $149(81.0)$ & 0.356 \\
\hline \multicolumn{8}{|l|}{ Hypertension } \\
\hline No & 275 & $7(2.5)$ & $26(97.5)$ & & $53(19.3)$ & $222(80.7)$ & \\
\hline Yes & 77 & $22(28.6)$ & $55(71.4)$ & $0.001 *$ & $20(26.0)$ & $57(74.0)$ & 0.103 \\
\hline \multicolumn{8}{|l|}{ BCG Vaccinated } \\
\hline No & 21 & $6(28.6)$ & $15(71.4)$ & & $15(71.4)$ & $6(28.6)$ & \\
\hline Yes & 331 & $23(6.9)$ & $308(93.1)$ & $0.002 *$ & $36(39.0)$ & $117(61.4)$ & $0.001 *$ \\
\hline
\end{tabular}

PDM- Pre-diabetes mellitus: Defined as glycated hemoglobin (HbAic) level 5.7-6.4 and never been diagnosed of diabetes mellitus.

LTBI-Latent Tuberculosis Infection: Defined as Quantiferon-TB Gold-In-Tube (QFT-GIT) Positive ( $\geq 0.35$ IU/ml) and never been diagnosed of Tuberculosis Kg-Kilogram

$* \mathrm{P} \leq 0.05$ was considered statistically significant. 
Table 4. Predictors of Concurrent PDM and LTBI.

\begin{tabular}{llll}
\hline Variable & Odds ratio & $\begin{array}{l}\text { Confidence } \\
\text { interval (95\%) }\end{array}$ & P value \\
\hline Age & 1.0 & & \\
Age $\leq 40$ years & 2.7 & $(2.25-8.85)$ & $0.0002^{*}$ \\
$\begin{array}{l}\text { Age }>40 \text { years } \\
\text { Smoking }\end{array}$ & 1.0 & & \\
No & 1.80 & $(1.51-6.32)$ & $0.001^{*}$ \\
Yes & & & \\
Family History of DM & 1.0 & $(1.67-17.92)$ & $0.001^{*}$ \\
No & 2.10 & & \\
Yes & 1.0 & $(1.9-2.7)$ & 0.102 \\
Family History of TB & & & \\
No & 2.5 & & \\
Yes & 1.0 & $(0.43-1.65)$ & 0.147 \\
Hypertension & 0.86 & & \\
No & & & \\
Yes & 1.0 & $(2.18-21.77)$ & $0.001^{*}$ \\
Non-BCG Vaccinated & & \\
No & 2.4 & & \\
Yes & & & \\
\hline
\end{tabular}

PDM - Pre-diabetes mellitus: Defined as glycosylated hemoglobin $\left(\mathrm{HbA}_{1 \mathrm{c}}\right)$ level between 5.7-6.4\% and never been diagnosed with diabetes mellitus.

LTBI - Latent Tuberculosis Infection: Defined as Quantiferon-TB-Gold-In Tube (QFT-GIT)

Positive $(\geq 0.35 \mathrm{IU} / \mathrm{ml})$ and never been diagnosed of Tuberculosis

BCG - Bacille-Calmette-Guerin

Odds Ratio of 1.0 represents the Reference group

$* \mathrm{P} \leq 0.05$ was considered statistically significant

\section{Discussion}

The association between type-2 diabetes mellitus (DM) and pulmonary tuberculosis (TB) is well-documented $(1,2$, $3,4,5,35$ ), and it is recognized that most individuals do not just develop DM and TB overnight, they go through prolonged intermediate phase of pre-diabetes (PDM) and latent tuberculosis infection (LTBI) respectively. However, there is paucity of data on PDM and LTBI from the DM- and $\mathrm{TB}$ laden countries to inform recommendations on intervention in those settings.

To our informed knowledge, this is the first study in Nigeria to systematically compare the prevalence of PDM among adults with or without LTBI. Unlike many previous studies in DM- and TB co-endemic populations, this work relied upon the use of standardized glycated hemoglobin $\left(\mathrm{HbA}_{1 \mathrm{c}}\right)$ quantitation and interferon-gamma (IFN- $\gamma$ ) production for the simultaneous measurement of PDM and LTBI respectively.

This multi-community study of apparently healthy adults in southern Nigeria showed overall prevalence of $29(8.2 \%)$ and $83(23.6 \%)$ for PDM and LTBI respectively, and identified a set of $9(2.6 \%)$ adults with concurrent PDM and LTBI (PDMLTBI). The study also demonstrated that a similar cluster of non-constitutive and constitutive host factors, including residence status, aging, smoking, familial diabetes as well as non-BCG vaccination were significantly associated with PDM and LTBI. Interestingly, this study revealed that the non-BCGvaccinated older-aged adults (50-59 years) were the most susceptible to PDM/LTBI and PDM-LTBI.
The prevalence rates for PDM and LTBI as observed in the present study is comparable to the findings in some previous studies. The prevalence of $8.2 \%$ for PDM in this study is similar to the reported $6.2 \%$ in Oke-Ogun region of Oyo state, Nigeria [32] and the $8.5 \%$ in Tamil-Nadu, India [33] but significantly different from the $13.8 \%$ recorded for periurban Uganda [34] and the 33.8\% observed among recentlyarrived refugees in Atlanta, USA [35]. The high prevalence of $23.6 \%$ for LTBI in this work is also similar to the reported rates in other African countries, which ranged from $31.2 \%$ in Ethiopia [36] to $49.0 \%$ in urban Uganda [37] and 55.2\% in South Africa [38]; while the American study reported 31.3\% [35]. It is noteworthy that it was only the American study that simultaneously screened for both PDM and LTBI and used standardized techniques as employed in the present work. However, that study involved refugees of all age categories who were originally from various low-middle-income countries, unlike the present study that specifically screened adults.

The site-specific prevalence for PDM and LTBI in this work showed striking variation between the different study sites (Figure 1). The highest prevalence of LTBI was observed among the rural dwellers who were at least twice as unlikely to develop PDM compared to the urbanites. The higher prevalence of LTBI found among the rural dwellers in this work could be attributed to their high crowding index, crowding in the village may be fuelling high Mycobacterium tuberculosis cross-infection among house inhabitants. The rural/semi-urban/urban variation in the prevalence of PDM and LTBI as found in the present work has justified the rationale for the sampling from three different communities, which assured representativeness of the southern Nigeria population and thereby enhancing the credibility of the results from this study.

The most important determinant discovered for susceptibility to PDM, LTBI or PDM-LTBI in this study is age. The results showed a dose-response relationship, with a three-fold increase observed in the prevalence of PDM and LTBI from aged 20-39 years up to aged 50-59 years, which was the age group identified to be almost four times more likely to be associated with dual PDM-LTBI state when compared to the younger 20-39 years old category. The increasing prevalence of these chronic conditions with aging in the present study is in consonance with the disorders of glucose metabolism being common in elderly people, and the accumulation of Mycobacterium tuberculosis over time in the TB-laden Nigerian communities as a result of the recognized waning effect of $\mathrm{BCG}$ vaccination with increasing age [39]; even though a high majority (94.0\%) of the studied adults had received the BCG vaccine. Taken together, this may denote age-dependency and a cumulative life-time risk of developing PDM and LTBI. This observation is also in concert with the concept of 'inflamm-aging', which describes an ever increasing chronic background of inflammation from sources such as senescent cells, cell debris and changes in the gut biota with age; that may then drive the immune system dysfunction [40]. 


\section{Study Limitations}

The main limitation to the current study is that it is cross-sectional and therefore causal relationship between risk determinant and the occurrence of PDM and/or LTBI could not be established. Some of the socio-demographic data may suffer from limitations of recall bias and stigmatization even though the WHO STEPS Wise approach, that was partially adapted in the current study, has been validated for similar chronic disorders in different populations. Moreover, this study is limited by the small sample size, which was necessitated by the limited resources available for studying in a resourceconstrained setting. However, the multi-community sampling from three (3) different and heterogeneous communities has ensured representativeness, enhancing the quality of the data from this work.

\section{Conclusion}

This multi-centric study of apparently healthy adults from southern Nigeria found a moderate prevalence of intermediate hyperglycemia (PDM) and high rate of Mycobacterium tuberculosis carriage (LTBI) among the adults, some of them even harbored PDM and LTBI concurrently. The data revealed that the prevalence of PDM among the adults with LTBI was more than twice the rate of those without it. This study observed that PDM was more common among the urbanites whereas LTBI prevails in the rural area. Finding such significantly high levels of these chronic disorders among presumably healthy, low-risk individuals is worrisome, more so as they were all unaware of their status until when diagnosed during this non-routine screening. It indicates that PDM and LTBI have become twin epidemics among the adult population in the study area. Nevertheless, this revelation presents a window of opportunity for targeted bidirectional screening for PDM and LTBI and priority allocation of scarce resources to the highest-risk population segments, with the aim of early detection and possible prevention or at least slowing down of the progression to full blown diseases and their potential complications. For a start, the nine (9) older-aged adults discovered in the course of the current work to be laden with dual PDM-LTBI will be monitored and recommended for intervention management as they are more likely to develop deregulated / impaired immunity.

This study observed that PDM and LTBI considerably overlap with respect to the clustering of certain constitutive and non-constitutive host factors. Aging was discovered as the most important independent determinant of PDM and /or LTBI. The age-dependent dosing effect observed in this study suggests that 'inflamm-aging' may be playing some role in susceptibility to dysfunctional/impaired immunity leading to the chronic state of deregulated glucose metabolism/carriage of Mycobacterium tuberculosis, that manifests in PDM and LTBI respectively.
The findings in the present study have highlighted the prevalence and identified some determinants of susceptibility to PDM and LTBI in southern Nigeria. The limitations observed in the study underline the need for additional large prospective studies in populations with similar backgrounds, with a view to a better understanding of the interrelationship between PDM and LTBI at both individual- and community levels, which will be important in the development of efficient and effective intervention programs in those high DM-and TB laden populations.

\section{References}

[1] Firanescu A-G, Popa A, Sandu M-M, Protasiewicz DA, Popa SG, Mota M. The global prevalence and incidence of diabetes mellitus and pulmonary tuberculosis. Roma J Diabetes Nutr Metab Dis 2016; 23 (3): 319-326.

[2] Viswanathan V, Kumpatla S, Aravindalochanan V et al. Prevalence of diabetes and prediabetes and associated risk factors among tuberculosis patients in India. PLoS One. e41367. doi: 10.371/journal. pone.0041367. 2012; 7.

[3] Dooley KE, Chaisson RE. Tuberculosis and diabetes mellitus: convergence of two epidemics. Lancet Infect Dis. 2009; 9: 937-74.

[4] Dixon B. Diabetes and tuberculosis: an unhealthy partnership. Lancet Infect. Dis 2007; 7: 444.

[5] Harries AD, Satyanarayana S, Kumar AMV et al. Epidemiology and interaction of diabetes mellitus and tuberculosis and challenges for care: a review. Public Health Action. doi: 10.5588/pha. 130024 PMID: 26393066. 2013; 13 [Suppl. 1] S3-S9.

[6] WHO / IUATLD Collaborative Framework for Care and Control of Tuberculosis and Diabetes. Geneva, Switzerland: WHO, 2011; WHO/HTM/TB/2011.15.

[7] Chatterjee R, Narayan KMV, Lipscomb J et al. Screening for diabetes and pre-diabetes should be cost-saving in patients at high risk. Diabetes Care. 2013; 36 (7): 1981-7.

[8] Petur Petursson. Aspects of Abnormal Glucose Regulation in Various Manifestations of Coronary Artery Disease. Coronary Artery Disease 2013; 24 (7): 577-582.

[9] Podell BK, Ackart DF, Kirk NM, Eck SP, Bell C, Basaraba RJ. Non-diabetic hyperglycemia exacerbates disease severity in Mycobacterium tuberculosis infected guinea pig. PLoS One. e46824. PubMed 23056469. 2012; 7.

[10] Tabak AG, Herder C, Rathman W, Brunner EJ, Kivimaki M. Prediabetes: A high-risk state for developing diabetes. Lancet. 2012; 379 (9833): 2279-2290.

[11] World Health Organization, International Diabetic Federation. Definition and diagnosis of diabetes mellitus and intermediate hyperglycemia: a report of a WHO/IDF Consultation. Geneva: WHO. 2006.

[12] The American Diabetic Association, European Association for the Study of Diabetes, International Federation of Clinical Chemistry and Laboratory Medicine and the International Diabetes Federation Consensus Statement on Worldwide Standardization of the Haemoglobin $\mathrm{A}_{1 \mathrm{c}}$ Measurement. Diabetes Care. 2007; 30: 2399-400. 
[13] Heianza Y, Hara $\mathrm{S}$, Arase $\mathrm{Y}$ et al. $\mathrm{HbA}_{1 \mathrm{c}}$ 5.7-6.4\% and impaired fasting plasma glucose for diagnosis of prediabetes and risk of progression to diabetes in Japan (TOPICS): a longitudinal cohort study. Lancet. 2011; 378: 147-155.

[14] Anjana RM, Shanthi Ram CS, Deepa M et al, Incidence of diabetes and pre-diabetes and predictors of progression among Asian Indians: 10-year follow-up of the Chennai Urban Rural Epidemiology Study (CURES). Diabetes Care. 2015; 38: 1441-1448.

[15] Almeida-Junior JL, Gil-Santana L, Oliveira CAM et al. Glucose metabolism disorder is associated with pulmonary tuberculosis in individuals with respiratory symptoms from Brazil. PLoS One. 2016; 11: 1-14.

[16] World Health Organization. Global Tuberculosis Report 2016. http://www.who.int/tb/publications/global_report.

[17] Vynnycky E, Fine PE. Lifetime risks, incubation period, and serial interval of tuberculosis. Amer J Epidemiol. 152: 247263.

[18] Mazurek GH, Jereb J, Vernon A et al. CDC updated guidelines for using Interferon gamma release assay to detect Mycobacterium tuberculosis infection-United States, 2010. MMWR Recommd. Rep. 2010; 59: 1-25.

[19] Diabetes Atlas Eight Edition Committee. IDF Diabetes Atlas: Brussels, Belgium: International Diabetes Federation. http://www.diabetesatlas.org/resources/2017.atlas.html. 2017.

[20] Global Tuberculosis Control 2018, WHO, Geneva. Use of high burden country list for TB by WHO in post-2015 era. $\mathrm{https} / / \mathrm{www}$,who.int/tb/publications/global_report/en/. 2018.

[21] Adeloye D, Ige JO, Aderemi AV et al. Estimating the prevalence, hospitalization and mortality from type 2 diabetes mellitus in Nigeria: a systematic Review and Meta-analysis. BMJ Open. e015424. doi: 10.2017; 7.

[22] Nigeria Bureau of Statistics. Annual Abstract of Statistics 2016-Proshare [cited July 19, 2017]. https://www.proshareng.com/news/General/NBS-AnnualAbsract-of-statistics....-/35588.

[23] Nigeria Diabetes Association Guidelines 2018. Published by the Diabetes Association of Nigeria. https//www.idf.org/ournetwork/regions-members/Africa.

[24] Nigeria National Tuberculosis and Leprosy and Buruli Ulcer Control Program (NTBLCP), National Strategic Plan-TB 2015-2020.

[25] World Health Organization. WHO STEPS Surveillance Manual. The WHO STEP Wise Approach to Chronic Disease Risk Factor Surveillance. Geneva, Switzerland. http://ww.whqlipdoc.who.int/publications/2005/9241593830eng.pdf. 2005.

[26] Meme N, Amwayi S, Nganga Z, Buregyeya E. Prevalence of undiagnosed diabetes and pre-diabetes among hypertensive patients attending Kiambu district Hospital, Kenya: a crosssectional study. Pan African Medical Journal. doi: 10.11604/pamj.2015.22.286.73. 2015; 22: 286.
[27] Ayah R, Joshi MD, Wanjiru R et al. A population-based survey of prevalence of diabetes and correlates in an urban slum in Nairobi, Kenya. BMC Public Health. 2013; 13: 371.

[28] Roche Cobas c111 User Manual. https://www.scribd.com/doc /9125231/Roche-cobas c111-user-Manual.

[29] Quantiferon-TB Gold (in-tube method). Package insert. www.Cellestis.com/IRM/company/show page. aspx (cpin1177). 2013.

[30] Akinshipe BO, Ezeani PC, Digban KA, Ehiaghe FA, Adedeji EB, Ehiaghe JI. Immunodiagnostic Potential of an In-vitro Interferon-Gamma Release Assay for Latent Tuberculosis Infection among Apparently Healthy Individuals in Okada Community, Nigeria. Int J Immunol. 2017; 5 (3): 41-48.

[31] Miri-Dashe T, Osewe S, Tokdung M et al. Comprehensive reference ranges for hematology and clinical chemistry parameters derived from normal Nigerian adults. PLoS One. doi: 10.1371/journal. pone, 0093919. 2014; 9 (5): 939.

[32] Rasaki SO, Kasali FO, Biliaminu SA, Odeigah LO. Prevalence of diabetes and pre-diabetes in Oke- Ogun region in Oyo State, Nigeria. J Medical Research and Health Education. 2017; 4 (1): 25.

[33] Muthunarayanan L, Ramraj B, Russel JK. Prevalence of prediabetes and its associated risk factors among rural adults in Tamil Nadu. Arch Med Health Sc. 2015; 3: 178-84.

[34] Chiwanga FS, Njelekela MA, Diamond MB et al. Urban and rural prevalence of diabetes and prediabetes and risk factors associated with diabetes in Tanzania and Uganda. Global Health Action. doi: 10.3402/ gha. V 9.31440. 2016; 9: 10.

[35] Hensel RL, Kempker RR, Tapia J, Oladele A, Blumberg HM and Magee MJ. Increased risk of latent tuberculosis infection among persons with prediabetes and diabetes mellitus. Int $\mathrm{J}$ Tuberc Lung Dis. doi: 10.5588/ijtld.15.0457. 2016; 20 (1): 7178.

[36] Legesse M, Ameni G, Mamo G et al. Performance of Quantiferon-TB Gold In Tube for the diagnosis of Mycobacterium tuberculosis (Mtb) infection in Afar Pastoralists, Ethiopia. BMC Infectious Diseases. 2010; 10: 354.

[37] Sekandi JN, List J, Luzze $\mathrm{H}$ et al. Yield of undetected tuberculosis and human immunodeficiency virus co-infection from active case finding in urban Uganda. Int J Tuberc Lung Dis. 2014: 18 (1): 13-19.

[38] Shanaube K, Hargreaves J, Fielding $\mathrm{K}$ et al. Risk factors associated with positive QuantiFERON -TB Gold In-Tube and Tuberculin skin tests results in Zambia and South Africa, PLoS One. e18206. e. 2011; 6 (4).

[39] Colditz GA, Brewer TF, Berkey CS et al. Efficacy of BCG vaccine in the prevention of Tuberculosis: Meta-analysis of the Published Literature. JAMA. 1994; 271: 698-702.

[40] Ventura MT, Casciaro M, Gangemi $\mathrm{S}$ and Buquicchio R. Immunosenescence in aging: between immune cells depletion and cytokines up-regulation. Clin Mol Allergy. 2017; 15: 21. 